\title{
Characterization and Deer-Repellent Property of Chrysophanol and Emodin from Sicklepod Weed
}

\author{
Ziming Yue1, Te-Ming Tseng1*, Marcus Lashley² \\ ${ }^{1}$ Department of Plant and Soil Sciences, Mississippi State University, Starkville, MS, USA \\ ${ }^{2}$ Department of Wildlife, Fisheries \& Aquaculture, Mississippi State University, Starkville, MS, USA \\ Email: *t.tseng@msstate.edu
}

How to cite this paper: Yue, Z.M., Tseng, T.-M. and Lashley, M. (2018) Characterization and Deer-Repellent Property of Chrysophanol and Emodin from Sicklepod Weed. American Journal of Plant Sciences, 9, 266280.

https://doi.org/10.4236/ajps.2018.92022

Received: December 19, 2017

Accepted: January 23, 2018

Published: January 26, 2018

Copyright $\odot 2018$ by authors and Scientific Research Publishing Inc. This work is licensed under the Creative Commons Attribution International License (CC BY 4.0).

http://creativecommons.org/licenses/by/4.0/

\begin{abstract}
Deer, particularly white-tailed deer (Odocoileus virginianus), damage row crops such as soybean (Glycine max L.) and are a perceived problem in the continental US. Currently, the only widely used technique to control deer from crop browsing is establishment of fences, which is expensive, labor intensive, and most of the time ineffective. Studies have shown that sicklepod, Senna obtusifolia (L.), contains anthraquinone derivatives, which in separate studies were shown to be toxic to cattle, rats, rabbits, and horses, and repel herbivores primarily birds. However, information of the deer-repelling property of anthraquinone in sicklepod is lacking. Field tests conducted at our Captive Deer Facility at Mississippi State University (MSU) confirmed the deer-repelling property of anthraquinone extracts from sicklepod. Soybean plants applied with control treatment (water) were browsed by deer, while plants applied with sicklepod anthraquinone extracts were avoided. Using chromatography techniques, we found the levels of anthraquinone derivatives (chrysophanol, emodin) in sicklepod plant parts in the order: root > fruit $>$ stem/leaf. Hydrolysis of water extracts of sicklepod seed produced high emodin concentration, suggesting emodin glycoside as the main form of anthraquinone glycoside in sicklepod seed. Deer-repelling compounds can be extracted in its pure form from sicklepod and applied on soybean to increase its repelling efficacy on deer, and at the same time protect soybean yields.
\end{abstract}

\section{Keywords}

Sicklepod, Weed, Anti-Herbivore, Deer-Repellant, Anthraquinone Derivative, Soybean, Deer Browsing 


\section{Introduction}

In North America, among the wildlife species, deer, particularly the white-tailed deer (Odocoileus virginianus), is responsible for $70 \%$ of crop losses caused by wildlife [1] [2], costing farmers up to $\$ 4.53$ billion each year [3]. Damages caused by deer have been reported in corn [4] [5] and soybean [1] [6] [7]. Current widely used techniques to control deer in row crops are establishment of fences and application of repellents [8] [9]. In general, fencing is expensive and labor intensive to install, and effectiveness of repellents depends on numerous factors, such as rainfall, which may dissolve repellents thus requiring reapplication [10]. Moreover, the deer may ignore the deer-repellent odor and feed on repellents when extremely hungry [10]. With increasing costs of agricultural production, the economic impact from reduced crop yield is very important, especially for smaller farms not financially prepared for poor harvest due to yield losses. It is therefore critical to protect crop yield against deer damage.

Sicklepod (Senna obtusifolia L.) is among the ten most troublesome weeds in the southern US, and soybean harvested in the southern US contain up to $5 \%$ sicklepod seeds [11]. Sicklepod seeds cause soybean quality degradation and need to be separated, thus resulting in large amounts of sicklepod seed side products [11]. Studies have shown sicklepod as a leading cause of livestock poisoning in the southern US [12] [13]; however, there is no information on the deer repelling property of sickelpod. Sicklepod belongs to Leguminoseae, genus Senna, tribe Casiinae, section Chamafistula, series Trigonelloideae, and is a common weed native to the southeast US and California. This species was often confused with Senna tora in the literature [11] [14], which has lost several phytochemical components possessed by $S$. obtusifolia [15]. Senna obtusifolia has seeds with transverse areoles, anthers with short beaks, and one extra foliar nectary between the lower leaflet pairs. Sicklepod is shown to contain anthraquinone derivatives [14] [16] [17] [18], which are proven as repellents of geese and other birds, rodents and rabbits [19] [20] [21] [22] [23]. Among sicklepod plant parts, seeds were found to contain the highest concentration of anthraquinone derivatives, at least eight, and represented $1 \%-2 \%$ of seed weight; other seed components included 5\% - 7\% fats, $14 \%$ - 19\% protein, and 66\% - 69\% carbohydrate [11] [14] [24] [25]. Sicklepod roots were reported to contain the second highest concentration of anthraquinone derivatives among sicklepod plant parts, including: islandicin, helminthosporin, chrysophanol, physcion, xantharin, 8-O-methylchrysophanol, obtusifolin, emodin, and aloe-emodin [18]. The purpose of this study is to explore the deer-repelling potential of sicklepod extracts and characterize the deer-repelling compounds present in sicklepod plant parts. The long-term goal is to use sicklepod seed side product in soybean production to develop a cost-effective deer repellent to protect soybean, a major crop in the US.

\section{Materials and Methods}

\subsection{Plant Materials}

Sicklepod plants were propagated in pots for six weeks in a greenhouse to harv- 
est enough plant tissues (leaf, stem, root and fruit) for all experiments listed below. Fresh plant materials were homogenized with mortar and pestle in liquid nitrogen, freeze dried (pressure set point: $0.02 \mathrm{mBar}$, FreeZone 12, Labconco), and put into separate glass bottles for further processing. The water content of the plant materials were measured by weighing before and after freeze drying. The measured water contents were $89 \%, 83 \%$ and $83 \%$ for sicklepod leaf, stem and root, respectively.

\subsection{Sample Preparation}

Deer feed on soybean and avoid sicklepod plants (M. Lashley, personal observation). Sicklepod is similar to soybean in leaf physical texture, but is still avoided by deer; hence, deer avoidance of sicklepod is not due to leaf physical texture, but differences in primary chemistry. Both sicklepod and soybean plants belong to Leguminosae family and share some similarity, the prominent difference is that sicklepod contains significant amount of anthraquinone derivatives, which were reported to be toxic to cattle, rats, rabbits and horses [12] [19] [26] [27] [28]. As chrysophanol, emodin and physcion were listed as main anthraquinone components in sicklepod seeds and roots [14] [18], they were selected as standards to estimate anthraquinone derivative concentrations. Anthraquinone derivatives can be detected at $254 \mathrm{~nm}$ with the diode array detector (DAD) [29], and by comparing chromatograms of sicklepod and soybean at $254 \mathrm{~nm}$, the deer-repelling compounds or anthraquinone derivatives should fall into differential peaks; hence, deer-repelling compounds (anthraquinone derivatives) of sicklepod can be easily identified. Three extractions were adopted: Extraction 1 was used for qualitative analysis; Extraction 2 was used for quantitative analysis; and Extraction 3 was used for testing on captive deer. All quantitative analyses were conducted in three replicates.

Extraction 1: Sicklepod plant powder of $0.10 \mathrm{~g}$ was weighted into a $1.5 \mathrm{~mL}$ tube, followed by addition of $1 \mathrm{~mL}$ methanol and $200 \mu \mathrm{L}$ chloroform. The tube was mixed for $20 \mathrm{~min}$ and centrifuged at $16,100 \times \mathrm{g}$ for $10 \mathrm{~min}$ at room temperature. The resulting supernatant was filtered $(0.2 \mu \mathrm{m}$ pore size) and air evaporated at room temperature overnight for high performance liquid chromatography (HPLC) and thin layer chromatography (TLC) analyses. Soybean seeds were extracted the same way as indicated above and analyzed using HPLC to identify differential peaks in comparison with sicklepod seed.

Extraction 2: Sicklepod plant part powder of $1.0 \mathrm{~g}$ was weighted into a $50 \mathrm{~mL}$ tube, followed by the addition of $20 \mathrm{~mL}$ methanol, $5 \mathrm{~mL}$ double deionized water, and $5 \mathrm{~mL}$ chloroform. The tube was mixed for $4 \mathrm{~h}$ and vacuum filtered by filter paper at room temperature. Ten $\mathrm{mL}$ of methanol was used to rinse the plant powder in the Buchner funnel. The combined filtrate was evaporated to complete dryness in water bath at $60^{\circ} \mathrm{C}$, and then dissolved by adding $3 \mathrm{~mL}$ methanol for HPLC and TLC analyses. HPLC was used to determine the anthraquinone derivative contents in the plant parts. 
Extraction 3: Sicklepod seeds were ground by Wiley Mill to $2 \mathrm{~mm}$, and $50 \mathrm{~g}$ of the meal was boiled in $1 \mathrm{~L}$ double deionized (DDI) water for three hours. The mixture was blended on a commercial mixer (Mixer Model 909-2, Hamilton Beach Inc., Washington, NC, USA) for 30 min followed by another 3 hour of boiling. The paste was then centrifuged in $50 \mathrm{~mL}$ tubes and vacuum filtered by filter paper at room temperature. The extract was further concentrated in water bath at $80^{\circ} \mathrm{C}$ until volume reached $250 \mathrm{~mL}$. For analysis of the sicklepod extract, $10 \mathrm{~mL}$ of the extract and $1 \mathrm{~mL}$ of $10 \%$ sulfuric acid was added into a test tube with a cap, heated on water bath for three days at $80^{\circ} \mathrm{C}$. After centrifugation, the supernatant was decanted and the pellet was freeze dried. The dried pellet was dissolved in $2 \mathrm{~mL}$ of methanol and used for HPLC analysis.

\subsection{High Pressure Liquid Chromatography (HPLC) Analysis}

An Agilent 1100 series HPLC (Agilent, Santa Clara, CA) was used to analyze anthraquinone derivatives in the extracts. The HPLC consisted of a DAD, an online vacuum degasser, a quaternary pump, an autosampler and a thermostatted column compartment. The Agilent Chemstation A.10.02 software with a spectral module (Agilent Technologies Inc., Wilmington, DE, USA) was used to process the data. Separation was achieved on an Alltech Adsorbsphere reverse phase C18 column (150 mm $\times 4.6 \mathrm{~mm}$, Dr. A. Maisch High Performance LC GmbH, Germany) with particle size $3 \mu \mathrm{m}$. Anthraquinone derivatives were detected at 254 $\mathrm{nm}$ [29], with a flow rate of $1 \mathrm{~mL} / \mathrm{min}$ and column temperature of $30^{\circ} \mathrm{C}$. Peaks were identified using standard compounds (chrysophanol and emodin). The injection volume of $5 \mu \mathrm{L}$ was used. Gradient elution program was developed as follows: Eluent was mixed by acetonitrile with water (modified with $0.2 \%$ acetic acid). Acetonitrile kept $40 \%$ for the first 8 minutes, followed by increasing to $70 \%$ from 8 minutes to 21 minutes, and then kept at $70 \%$ to 25 minutes. Sicklepod plant part extracts (stem, root, fruit and leaf) from Extraction 2 were analyzed for chrysophanol and emodin.

\subsection{Thin Layer Chromatography (TLC)}

Thin layer chromatography was carried out on TLC plates (F254, Merck, Darmstadt, Germany) using eluents as follows: Benzene:methanol $=4: 1$. Methanol based extracts from sicklepod stem, root, and fruit were spotted on the TLC plates using a capillary pipette. The above eluent was used to develop in a sealed chamber. When the development front reached $1 \mathrm{~cm}$ below the top edge, the plate was taken out and air dried. Separated components were visualized under UV light at $302 \mathrm{~nm}$ (Variable Intensity UV Transilluminator, VWR Scientific, West Chester, PA, USA). Anthraquinone derivatives showed yellow/orange color. All experiments were conducted in three replicates. TLC allows a quick visualization of anthraquinone derivatives in situ within the plant parts on the basis of its UV fluorescence.

\subsection{UV Fluorescence of Anthraquinone Derivatives}

A fluorescence microscope (Olympus BX51) equipped with a cube consisting of 
filters D360/40x, 400dclp, and ET560lp, was used to observe the fluorescence of the anthraquinone derivatives. The latter filter completely blocks the UV excitation $(350 \mathrm{~nm})$ and allows only signals of approximately $560 \mathrm{~nm}$ and longer to be passed. The UV excitation of the microscope was $350 \mathrm{~nm}$. Such setting allowed the microscope to observe fluorescence of anthraquinone derivatives around 580 - $600 \mathrm{~nm}$ (yellow). Fresh plant tissues (sicklepod root, stem and soybean stem) were cut free-hand using a razor blade into thin sections and immediately examined under the microscope. All observations were in three replicates.

\subsection{Captive Deer Test}

The captive deer test were conducted in the captive deer facility at Mississippi

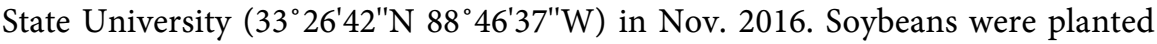
at a density of 516 plants $/ \mathrm{m}^{2}$ in plastic trays $(11 \times 21 \times 2.5 \mathrm{in})$ filled with potting soil (Sunshine Mix \#1; Sun Gro Horticulture Inc., Bellview, WA, USA), and propagated in a greenhouse at RR Foil Plant Science Research Center, Mississippi State University. Once plants reached V2 stage the soybean seedling in each tray was either sprayed with $200 \mathrm{~mL}$ sicklepod seed extract (Extraction 3) or water (control), using a $1 \mathrm{~L}$ spray bottle. Three replicate trays for each treatment was used. Trays were then moved into a $18,000 \mathrm{sq} f t$ deer enclosure in the captive deer facility containing four captive male deer, and arranged in a completely randomized design inside the enclosure. The soybean seedling trays were exposed to deer browsing for 4 hours. Images of soybean seedling before and after 4 hour deer exposure was captured using a Nikon D7000 digital camera (Nikon, Tokyo, Japan) and analyzed using image analysis software Image J (US National Institutes of Health, Bethesda, MD, USA) to determine percent browsing. Browsing was determined by reduction in canopy cover, which was estimated using green leaf pixels and total number of pixels, and was calculated using the formula: Canopy cover $(\%)=100 \times$ (green pixel count)/(total pixel count). Visual estimates of deer browsing (visual browsing) of soybean plants in each tray was also recorded and ranged from $0 \%$ to $100 \%$, where $0=$ no browsing, and 100 = completely browsed.

\subsection{Reagents and Stock Solutions}

HPLC grade water and acetonitrile were from Burdick \& Jackson (Muskegon, MI, US); acetic acid was from Fisher Scientific (Hampton, NH, US); methanol was from BDH VWR Analytical (Radnor, PA, US). Emodin (98\%) was from BioVision (San Francisco, CA, US); Chrysophanol (95\%) was from TCI America (Portland, OR, US). Emodin and chrysophanol (2.0 mg each) were weighed into $100 \mathrm{~mL}$ volumetric flasks, respectively. Methanol was added to the flasks, heated to dissolve, and then filled to marks with methanol after cooling down. The final concentration of both emodin and chrysophanol was $20 \mathrm{mg} / \mathrm{L}$.

\subsection{Data Analysis}

HPLC analyses including extractions were conducted in three replicates using 
external standards. Peak heights of standards 20,10,5, 1, 0.5, $0.25 \mathrm{mg} / \mathrm{L}$ were used to make calibration curves $\left(\mathrm{R}^{2}>99.9 \%\right)$. Relative standard deviation (RSD) for retention time was less than $0.5 \%$ and RSD for athraquinone derivatives were less than $7 \%$ based on five injections. Data (HPLC and captive deer test) were analyzed by Student's t-test using the software JMP 13.0.0, statistical significant level was taken at $\mathrm{p}<0.05$. Standard deviation error bars and letters indicating statistical differences were obtained from the analyses.

\section{Results}

\subsection{Comparison between Soybean and Sicklepod Compositions}

As deer prefer soybean rather than sicklepod, the deer-repelling compounds of sicklepod should be associated with compounds that are present exclusively in sicklepod. Figure 1(A) shows the chromatogram comparison between soybean and sicklepod seeds at $254 \mathrm{~nm}$ detection wavelength (Extraction 1). The retention times (RT) at which the peaks between sicklepod and soybean seeds were significantly different were 16.08 and $21.02 \mathrm{~min}$. Seed was used for this purpose for more pronounced differential peaks. These two peaks may correspond to the presence of two deer repelling compounds in sicklepod seeds which are absent in soybean seeds.

\subsection{Identification of the Deer Repelling Compounds in Sicklepod}

Extraction 1 and contrast between soybean and sicklepod were used to locate the deer repelling compounds in sicklepod seeds. As chrysophanol and emodin are the most abundant anthraquinone derivatives in sicklepod seeds [14], the above pronounced differential peaks are suspected to be chrysophanol and emodin. Mixed standards of emodin and chrysophanol were run on the HPLC with same conditions as mentioned previously (Figure 1(B)). Based on the standard retention times, the deer repelling compounds at RT $16.08 \mathrm{~min}$ and $21.02 \mathrm{~min}$ (Figure 1(A)) were confirmed to be emodin and chrysophanol, respectively.

\subsection{Distribution of Deer-Repelling Compounds in Different Parts of Sicklepod Plant}

The extracts of different plant parts from Extraction 2 were analyzed to calculate the deer-repelling compound concentrations in sicklepod plant parts. The calculated chrysophanol and emodin contents in leaf, stem, root and fruit are plotted in Figure 2. Sicklepod fruit and root showed significantly higher anthraquinone derivative (chrysophanol and emodin) contents than stem and leaf, with the stem and leaf having similar anthraquinone derivative contents (Figure 2). In sicklepod, the root is dominated by chrysophanol $(125 \mu \mathrm{g} / \mathrm{g})$ while the fruit contains both chrysophanol and emodin at similar, but high levels (42 and 44 $\mu \mathrm{g} / \mathrm{g}$ respectively). A chromatogram comparison between sicklepod stem and root indicates the root having higher chrysophanol and emodin concentrations than the stem (Figure $1(\mathrm{C})$ ). 


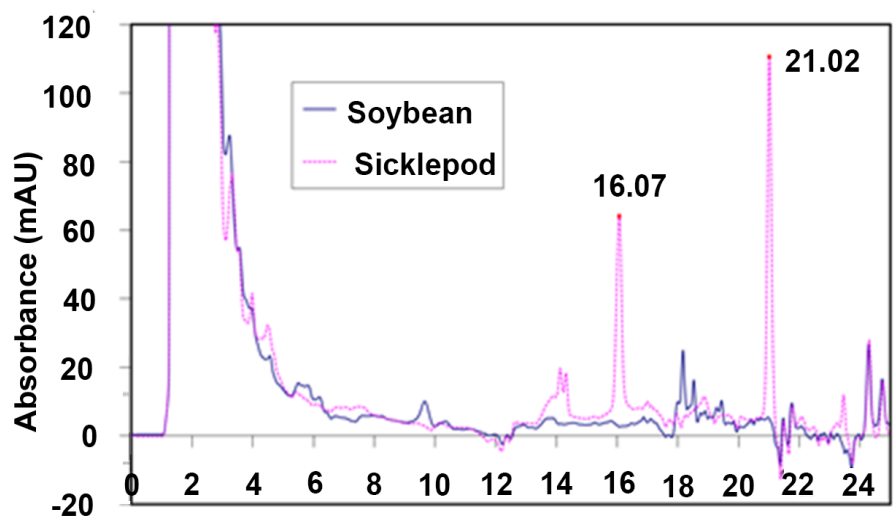

Retention time (min)

(A)

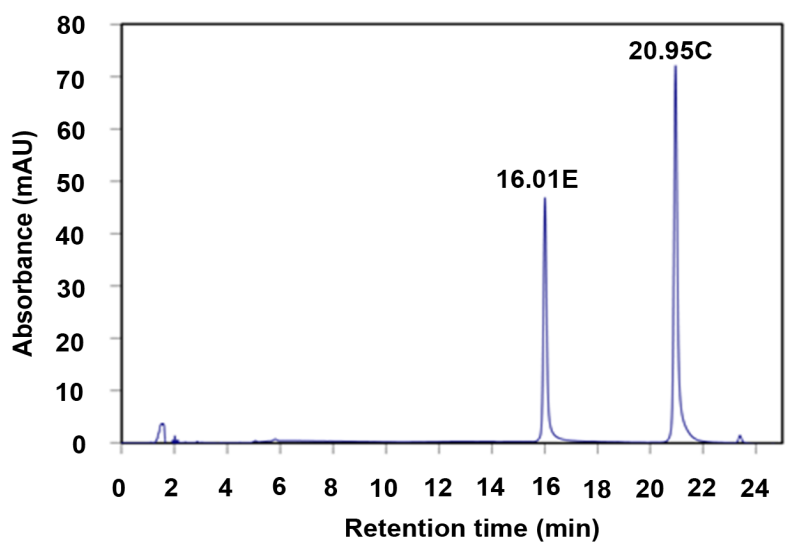

(B)

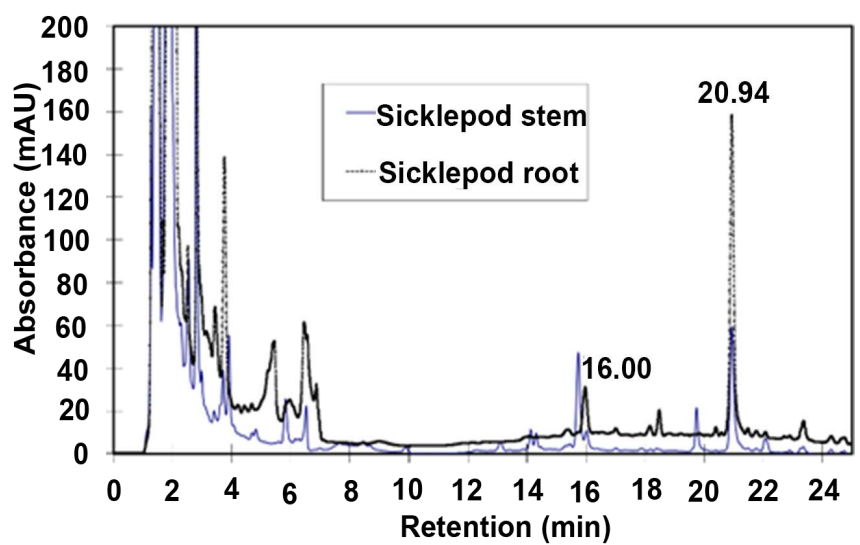

(C)

Figure 1. (A) Chromatogram comparison between sicklepod and soybean seeds; the most pronounced differential peaks were found at retention time (RT) 16.07 and $21.02 \mathrm{~min}$; (B) Chromatogram of mixed standards of chrysophanol and emodin, their RT 16.01 and $20.95 \mathrm{~min}$, respectively, match with the differential peaks in (A); (C) Chromatogram comparison between sicklepod stem and root. The root showed higher concentrations of chrysophanol and emodin.

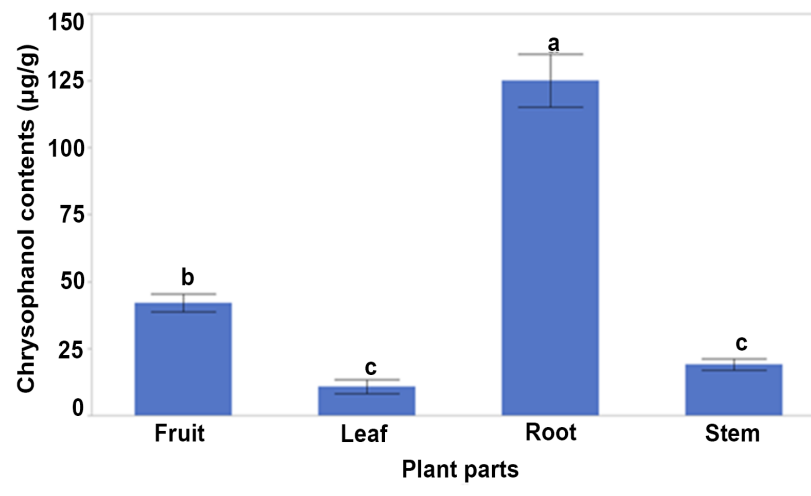

(A)

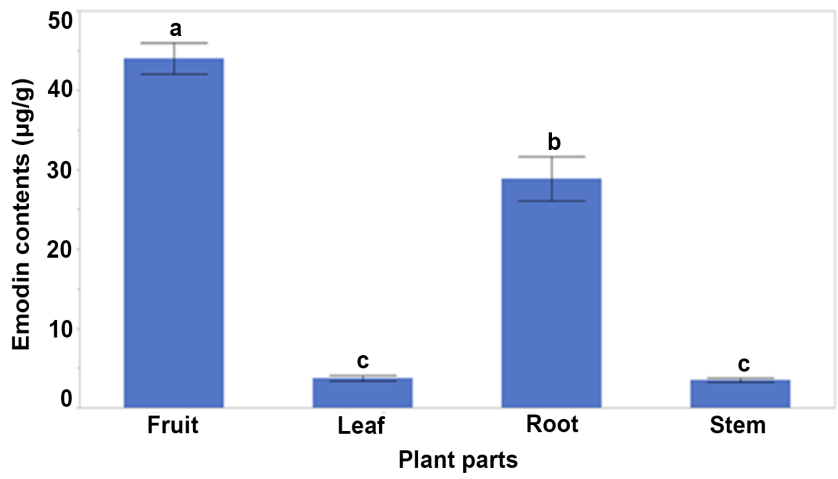

(B)

Figure 2. Anthraquinone derivative content comparison in different sicklepod plant parts: (A) Chrysophanol: sicklepod root and fruit have higher chrysophanol contents than stem and leaf; (B) Emodin: sicklepod fruit and root have higher emodin contents than stem and leaf. Statistical letters a, b and $c$ were obtained from JMP 13.0.0; means with different letters are significantly different at $\mathrm{p}<0.05$. 


\subsection{Thin Layer Chromatography (TLC)}

Methanol extracts of sicklepod plant parts primarily contain anthraquinone derivatives and these compounds can be viewed analytically using TLC. The TLC development plate (Figure 3) containing sicklepod stem, root, and fruit extracts (Extraction 2) indicate that most of the anthraquinone derivatives are present in the top half of the development plate with chrysophanol at the top-most and emodin in the middle (Figure 3). The fruit extract shows the presence of bright bands near the original spot suggesting the presence of anthraquinone derivative glycosides. The results also indicate that sicklepod fruit, stem and root contain more than two anthraquinone derivatives (chrysophanol and emodin).

\subsection{Anthraquinone Visualization under UV Fluorescence}

The UV fluorescence of anthraquinone derivatives in sicklepod extracts from TLC was observed. These compounds also have fluorescence in the plant in situ. The UV fluorescence images of sicklepod stem show the stem bark having lower fluorescence suggesting lower content of anthraquinone derivatives (Figure 4(A) and Figure 4(B)). The fluorescence microscope images of sicklepod root and stem, and soybean stem (Figures $4(\mathrm{C})-(\mathrm{E})$, respectively) indicate all the plant surfaces have low fluorescence thus suggesting lower anthraquinone derivative contents on the outer compared to the inner layers of the plant. Sicklepod stem can be divided into three parts: pith, cortex and bark, and their anthraquinone derivative content, decrease in this same order, based on their UV fluorescence.

\subsection{Captive Deer Test}

The field test at the captive deer facility was conducted to test the efficacy of the sicklepod extract in repelling deer. Results show significant contrast between

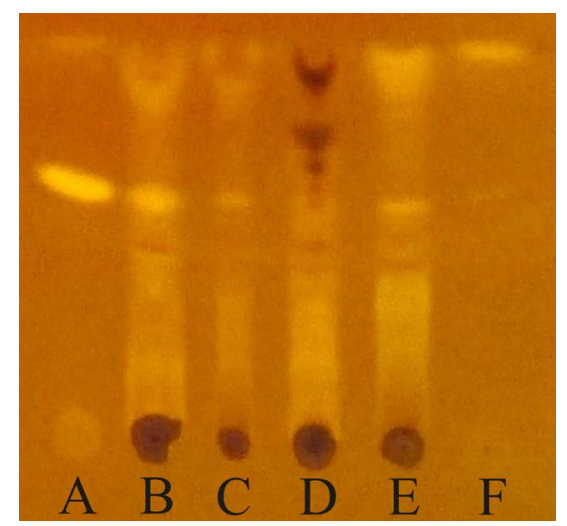

Figure 3. From left to right: (A) emodin standard; (B) sicklepod fruit extract; (C) sicklepod stem extract; (D) sicklepod leaf extract; (E) sicklepod root extract; and (F) chrysophanol standard. Solvent: Benzene:methanol $=4: 1$. The yellow fluorescence of emodin and chrysophanol occurred in sicklepod fruit, root and stem. These anthraquinone derivatives were relatively less in sicklepod leaves. 


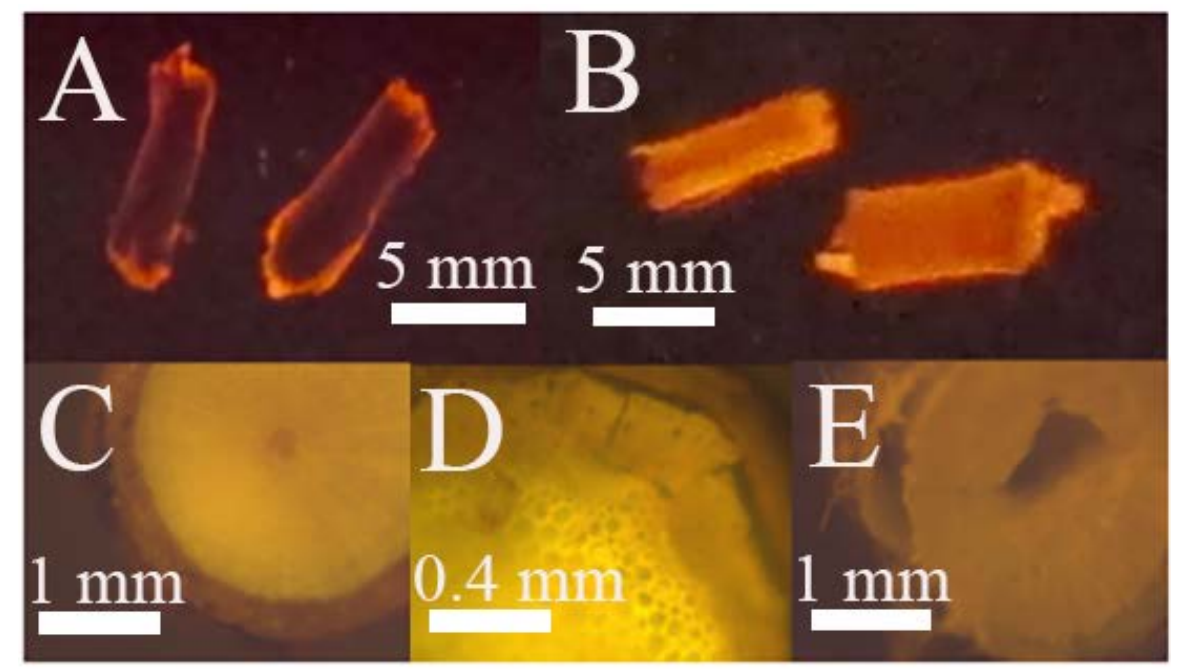

Figure 4. UV Fluorescence microscope images of sicklepod and soybean plant parts: Sicklepod stem with bark (A) and without bark (B) indicate lower anthraquinone derivatives in the bark. Sicklepod root section (C) show bark having lower anthraquinone derivative contents than the inside. Sicklepod stem section (D) show bark having lower anthraquinone derivative contents and decrease in the order of pith, cortex and bark. Soybean stem section (E) show lower anthraquinone derivative contents (less fluorescence) than sicklepod.

soybean plants treated with water (control) and sicklepod extracts (Figure 5). Their browsing rates were determined on the basis of canopy reduction (\%) and visual browsing (\%). Soybean plants applied with water showed 92\% reduction in canopy cover, while plants applied with sicklepod extract only showed $23 \%$ reduction in canopy cover, thus supporting the ability of sicklepod extract in re pelling deer and protecting soybean plants from being browsed. Visual browsing of water and sicklepod extract treated soybean plants were $92 \%$ and $18 \%$, respectively. The result is consistent with the canopy reduction analysis (Figure 5). The sprayed extract was hydrolyzed and analyzed for anthraquinone derivatives present. The results showed high emodin $(40 \mu \mathrm{g} / \mathrm{mL})$ and relatively low chrysophanol concentrations (below $5 \mu \mathrm{g} / \mathrm{mL}$ ) in the sicklepod extract used in the captive deer test.

\section{Discussions}

\subsection{Comparison between Sicklepod and Soybean Deer-Repelling Mechanism}

The quantification of anthraquinone derivatives in different parts of sicklepod plant indicates the fruit containing the highest anthraquinone derivative content among other plant parts. Similarly, another study showed that sicklepod seeds contained anthraquinone derivatives up to $2 \%$ [14]. Sicklepod fruits occur relatively early (fourth week after emergence) after blooming, and the pods always project on the outer side of the plant body (Figure 6). The out-extended fruits (high anthraquinone derivative contents) possibly act as a defense mechanism 


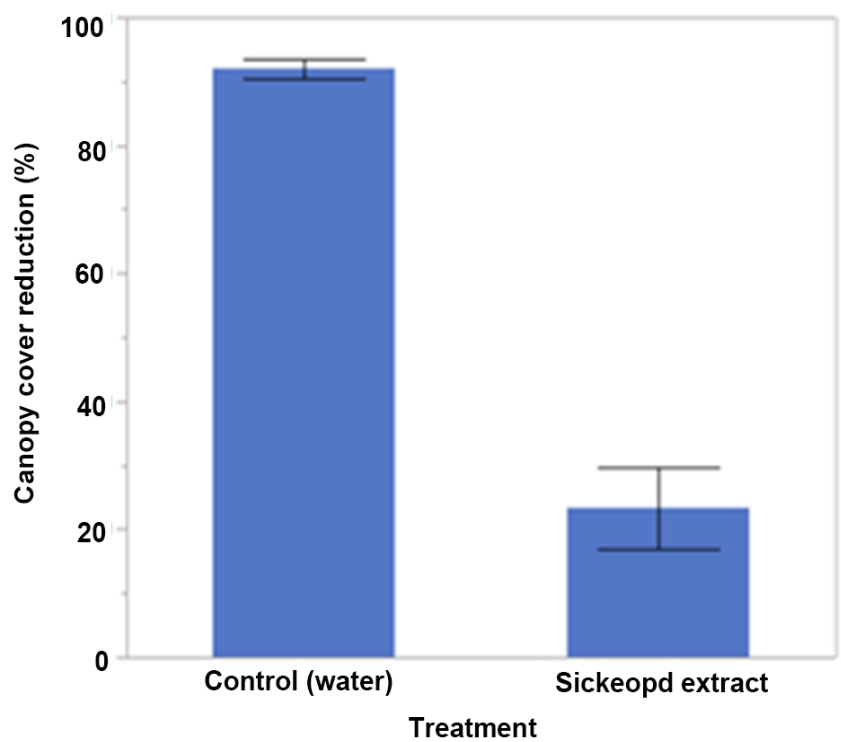

(A)

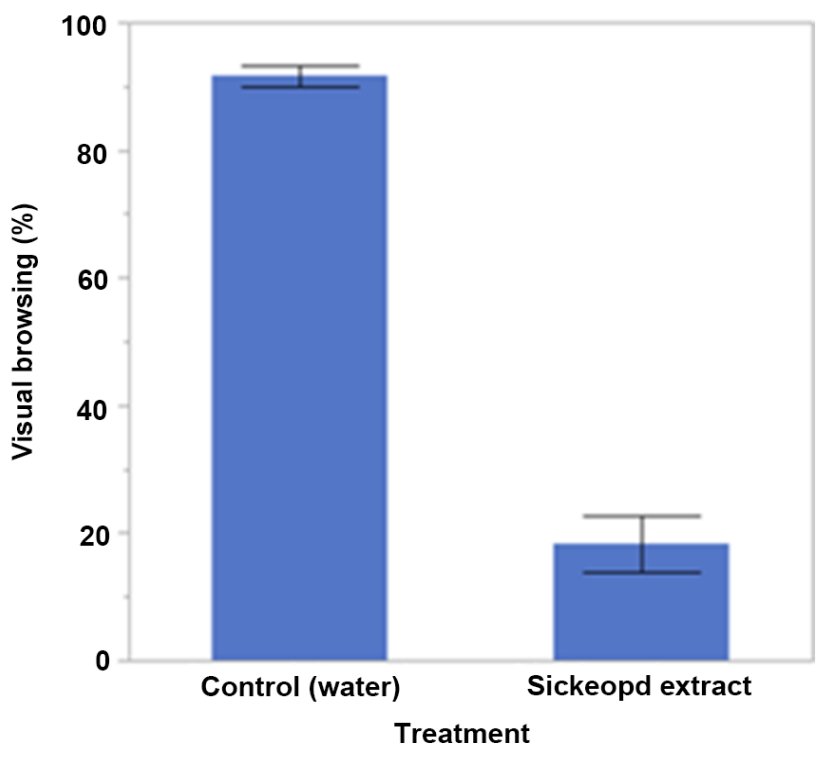

(B)

Figure 5. Canopy cover reduction (\%) (A), and visual browsing (\%) (B) of soybean seedlings treated with water (control), and sicklepod seed extract, after $4 \mathrm{hr}$ of exposure to captive deer at the captive deer facility at Mississippi State University.

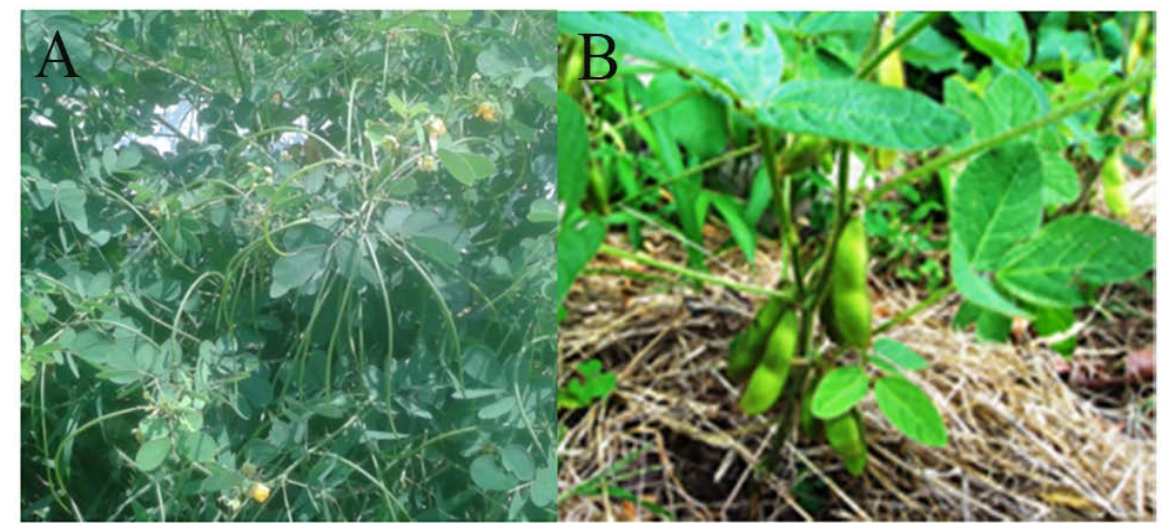

Figure 6. Sicklepod plant (A) and soybean plant (B) comparison shows sicklepod fruits projecting out (defending) while soybean fruits hang from the auxiliary leaf locations (protected). Sicklepod fruits contain high anthraquinone derivative contents in their endosperm, while soybean fruits contain minimal deer-repelling compounds and no endosperm in the mature seeds.

against herbivores such as deer from leaf browsing. When the leaves are being browsed, the out-extended fruits are difficult to avoid. Such defense mechanism may also help in seed transport and germination, as consumption by herbivores may scarify the seed during chewing (hard seed coat) and help the seed germinate and being transported after excretion, if the seed is not digested [30]. On the other hand, soybean fruits usually hang from auxiliary leaf positions (protected by leaves) (Figure 6). And, because soybean seeds contain low anthraquinone contents, both the leaves and pods are more likely to be browsed by deer. 


\subsection{Anthraquinone Concentrations in Different parts of Sicklepod Plant}

Results from this study show that anthraquinone derivative concentrations at different parts of sicklepod plant were in the order: root $>$ fruit $>$ stem/leaf, and this is consistent with previous results [17]. In addition, when sicklepod stems, with and without bark, were viewed under the UV transilluminator, stems without bark fluoresced bright yellow, while stems with bark were dark except at the two ends (Figure 4(A) and Figure 4(B)). The UV source $(302 \mathrm{~nm}$ ) was at the bottom and because stems are not transparent to UV, the yellow fluorescence from the stems without bark was excited by UV reflected from the chamber. The fluorescence microscope images confirmed that the bark (epidermis cells) of the stem had lower florescence than the pith, thus implying that bark has lower anthraquinone derivative content. Similarly, the sicklepod leaf surface (upper and lower epidermis) was shown to contain lower fluorescence than the inside (mesophyll cells) (image not provided). All plant surfaces (except root cap) viewed under the UV were observed to have lower florescence than the inside. Han et al. [31] noted that the absence of light significantly enhanced production of anthraquinone derivatives from Rubiceae plant cell cultures. Sicklepod (Leguminosae family) anthraquinone biosynthesis follows a different pathway from plants belonging to Rubiaceae family [31]. However, our observation shows its anthraquinone biosynthesis follows the same rule mentioned above: light avoidance. Another possibility of the above observation is that anthraquinone derivatives may not be light stable. The biosynthesized anthraquinone derivatives may decompose in presence of light; however, this is not true since Vanderveer [32] found anthraquinone derivatives (insecticide) to be light stable. The light avoiding nature of anthraquinone derivative biosynthesis in sicklepod plants may also be the reason why these compounds are present in high amounts in roots, where light is absent.

\subsection{Anthraquinone form Stored in Sicklepod Plant}

Anthraquinone derivatives in sicklepod plants mainly exist in glycoside form (around 90\% of total anthraquinone derivatives), and only minor amount of anthraquinone derivatives are in free anthraquinone form (aglycones) [14] [16] [17]. This information is important because it indicates that the glycoside form of anthraquinone derivatives can be extracted using water since they are water soluble. The water soluble portion of anthraquinone derivatives may also allow these secondary metabolites to be transported in the plant body, as compared to free anthraquinones (aglycones) that are insoluble in water and difficult to be transported within the plant. Both, glycosides and free anthraquinones, may act as effective components associated with deer-repelling property in sicklepod. The hydrolyzed sicklepod seed extract contained higher emodin concentration $(40 \mu \mathrm{g} / \mathrm{mL})$ suggesting anthraquinone glycosides in the extract being dominated by emodin glycoside. 


\subsection{Superior than Tannin as Deer-Repelling Compounds}

Besides anthraquinone derivatives (emodin and chrysophanol, etc.) and their glycosides, tannin was reported to be a component of sicklepod leaves $(0.5 \%)$ [33] and seeds (0.4\%) [34], and was also thought to have deer-repelling property [35] [36]. Monteith [36] (2012) tested tannin as a deer repellent, and the applied tannin concentrations of $3 \%, 5 \%, 10 \%, 20 \%$ was much higher than tannin concentration naturally present in sicklepod leaves [33]. Compared to the tannin test range of $3 \%$ to $20 \%(\mathrm{w} / \mathrm{v})$ as a repellent, the tannin contents in sicklepod leaves and seeds is only $0.5 \%$ and $0.4 \%$, respectively. In addition, tannin was not limited to sicklepod, it was also contained in soybean meal (2.5\%) [37]. As is well known, soybean meal is a general food and fodder for humans and mammals; hence, the extremely low tannin concentrations in sicklepod leaves $(0.5 \%)$ and seeds $(0.4 \%)$ may not be significant enough to function as a deer repellent. Furthermore, tannin was thought as a deer-repelling compound because it imparted an astringent taste. Its repellent effect focused on the consumption phase (taste) [35], which is in contrast to anthraquinone derivatives, thought as a conditioned aversion deer repellent [10] where deer learned the toxic effect of anthraquinones during the digestion phase [38]. Additionally, a study by Monteith [36] (2012) showed that $10 \%(\mathrm{w} / \mathrm{v})$ tannin is required to achieve $72 \%$ reduction in deer browsing. In contrast, the effective concentration of anthraquinone derivatives to achieve $100 \%$ reduction in deer browsing was only around $0.01 \%(\mathrm{w} / \mathrm{v})$; this concentration is around 3 orders lower than tannin repellents.

\section{Conclusions}

The deer-repelling property of sicklepod is mainly associated with anthraquinone derivatives. Anthraquinone derivatives in sicklepod seed occur mainly as glycosides, comprising about $90 \%$ of total anthraquinone derivatives, while the remaining anthraquinone derivatives exist as free form [14]. Chrysophanol and emodin are the primary deer-repelling compounds identified in this study, and among the different parts of sicklepod plant, the total anthraquinone derivative concentration decreased in the order of root $>$ fruit $>$ stem/leaves. One reason why the root contains high levels of anthraquinone derivatives may be due to the absence of light, which promotes the biosynthesis of anthraquinone derivatives. UV fluorescence of anthraquinone derivatives allows us to view the contrast between soybean and sicklepod plants, and also anthraquinone distribution in sicklepod plant. This is a convenient way to observe where the anthraquinone is located in the sicklepod plant before proceeding with the complex and time consuming extraction and analysis.

The current work forms a basis for us to further apply the sicklepod deerrepelling property (anthraquinone derivatives as effective components) to protect soybean plants from injury and consequent yield reduction arising from deer grazing. We are currently testing the efficacy of different liquid formulations of sicklepod extracts from different parts of the plant on its ability to repel 
deer, by conducting over-the-top application of extracts on soybean plants, in a larger scale in field. Sicklepod formulations having the ability to repel deer may potentially be used as a natural deer repellent to protect the soybean yield and quality.

\section{Acknowledgements}

Funding for this project was provided by the Mississippi Soybean Promotion Board and the Mississippi State University Cross College Grant. This publication is a contribution of the Mississippi Agricultural and Forestry Experiment Station, and is based upon work that is supported by the National Institute of Food and Agriculture, US Department of Agriculture, Hatch project under accession number 230060. The authors would also like to express appreciation for the assistance in the field provided by Gourav Sharma, Swati Shrestha, Shandrea Stallworth, and Dan Morina.

\section{References}

[1] Begley-Miller, D.R. and Cady, A.B. (2015) White-Tailed Deer Browsing of Soybeans Significantly Changes Plant Morphology and Reduces Yield, Contributing to Large Financial Losses. Ohio Journal of Science, 115, 56-61. https://doi.org/10.18061/ojs.v115i2.4750

[2] NJAES (1998) How Are White-Tailed Deer Affecting Agriculture in New Jersey? https://njaes.rutgers.edu/pubs/deerdamage/

[3] Conover, M.R. (2002) Resolving Human-Wildlife Conflicts: The Science of Wildlife Damage Management. Lewis Publishers, Boca Raton.

[4] Wywialowski, A.P. (1996) Field Corn Lost to Wildlife in 1993. Wildlife Society Bulletin, 24, 264-271.

[5] Tzilkowski, W.M., Brittingham, M.C. and Lovallo, M.J. (2002) Wildlife Damage to Corn in Pennsylvania: Farmer and On-the-Ground Estimates. Wildlife Society Bulletin, 66, 678-682. https://doi.org/10.2307/3803134

[6] Tanner, G. and Dimmick, R.W. (1983) An Assessment of Farmers' Attitudes toward Deer and Deer Damage in West Tennessee. Proceedings of the Eastern Wildlife Damage Conference, 1, 195-199.

[7] Wallace, S.U., Palmer, J.H., Barnes, J.M., Francoeur, L.C. and Yarrow, G.K. (1996) Strategies for $\mathrm{r}$ Reducing Deer Damage to Soybeans. Clemson Univ. PSA Publication.

[8] Ver Cauteren, K.C., Lavelle, M.J. and Hygnstrom, S. (2006) Fences and DeerDamage Management: A Review of Designs and Efficacy. USDA National Wildlife Research Center-Staff Publications, 99. http://digitalcommons.unl.edu/icwdm_usdanwrc/99 https://doi.org/10.2193/0091-7648(2006)34[191:FADMAR]2.0.CO;2

[9] Wagner, K.K. and Nolte, D.L. (2001) Comparison of Active Ingredients and Delivery Systems in Deer Repellents. USDA National Wildlife Research Center-Staff Publications, 598. http://digitalcommons.unl.edu/icwdm_usdanwrc/598

[10] Ward, J.S. and Williams, S.C. (2010) Effectiveness of Deer Repellents in Connecticut. Human-Wildlife Interaction, 4, 56-66.

[11] Abbott, T.P., Vaughn, S.F., Dowd, P.F., Mojtahedi, H. and Wilson, R.F. (1998) Po- 
tential Uses of Sicklepod (Cassia obtusifolia). Industrial Crops and Products, 8, 77-82. https://doi.org/10.1016/S0926-6690(97)10010-3

[12] McCormack, J.E. and Neisler, W.E. (1980) Cassia obtusifolia Toxicity in a Dairy Herd. Veterinary Medicine, Small Animal Clinician, 75, 1849-1851.

[13] Mudge, D. (2010) Toxic Plants Recent Farm Animal Poisonings. Farm/Ranch Case Studies Series Fact Sheet \#032710.

[14] Crawford, L., McDonald, G.M. and Friedman, M. (1990) Composition of Sicklepod (Cassia obtusifolia) Toxic Weed Seeds. Journal of Agriculture and Food Chemistry, 38, 2169-2175. https://doi.org/10.1021/jf00102a014

[15] Upadhyaya, S.K. and Singh, V. (1986) Phytochemical Evaluation of Cassia obtusifolia L. and Cassia tora L. Proceedings of Indian Academy of Sciences, 96, 321-326.

[16] Koshioka, M. and Takino, Y. (1978) Studies on the Evaluation of Crude Drug (I). Chemical and Pharmaceutical Bulletin, 26, 1343-1347. https://doi.org/10.1248/cpb.26.1343

[17] Koshioka, M., Ikemoto, C., Nishimura, M., Ishii, Y. and Takino, Y. (1979) Studies on the Evaluation of Crude Drug (IV) Quantitative Estimation of Anthraquinone in Cassia Seeds. Shoyakugaku Zasshi, 33, 72-76.

[18] Kitanaka, S. and Takido, M. (1986) Studies on the Constituents in the Roots of Cassia obtusifolia L. and the Antimicrobial Activities of Constituents of the Roots and the Seeds. Yakugaku Zasshi, 106, 302-306. https://doi.org/10.1248/yakushi1947.106.4_302

[19] Nicholson, S.S., Flory, W. and Ruhr, L.P. (1985/86) Sicklepod Poisoning in Cattle: A New Development. Louisiana Agriculture, 29, 18-19.

[20] Avery, M.L., Humphrey, J.S. and Decker, D.G. (1997) Feeding Deterrence of Anthraquinone, Anthracene, and Anthrone to Rice-Eating Birds. Journal of Wildlife Management, 61, 1359-1365. https://doi.org/10.2307/3802138

[21] Werner, S.J., Carlson, J.C., Tupper, S.K., Santer, M.M. and Linz, G.M. (2009) Threshold Concentrations of an Anthraquinone-Based Repellent for Canada Geese, Red-Winged Blackbirds, and Ring-Necked Pheasants. Applied Animal Behaviour Science, 121, 190-196. https://doi.org/10.1016/j.applanim.2009.09.016

[22] Deliberto, S.T. and Werner, S.J. (2016) Review of Anthraquinone Applications for Pest Management and Agricultural Crop Protection. Pest Management Science, 72, 1813-1825. https://doi.org/10.1002/ps.4330

[23] Werner, S.J., DeLiberto, S.T., Baldwin, R.A. and Witmer, G.W. (2016) Repellent Application Strategy for Wild Rodents and Cottontail Rabbits. Applied Animal Behaviour Science, 185, 95-102. https://doi.org/10.1016/j.applanim.2016.10.008

[24] Harry-O'kuru, R.E. (2007) Scale-Up of Sicklepod Processing. In: Janick, J. and Whipkey, A., Eds., Issues in New Crops and New Uses, ASHS Press, Alexandria, 132-137.

[25] Kang, W., Yu, H. and Wang, J. (2012) $\alpha$-Glucosidase Inhibitory Compounds from Seeds of Cassia obtusifolia. Chemistry of Natural Compounds, 48, 465-466. https://doi.org/10.1007/s10600-012-0275-4

[26] Voss, K.A. and Brennecke, L.H. (1991) Toxilogical and Hematological Effects of Sicklepod (Cassia obtusifolia) Seeds in Sprague-Dawley Rats: A Subchronic Study. Toxicon, 29, 1329-1336. https://doi.org/10.1016/0041-0101(91)90119-C

[27] Putnam, M.R., Boosinger, T., Spano, J., Wright, J. and Wiggins, A. (1988) Evaluation of Cassia obtusifolia (Sicklepod) Seed Consumption in Holstein Calves. Veterinary and Human Toxicology, 30, 316-318. 
[28] Furlan, F.H., Zanata, C., Damasceno, E.S., Oliveira, L.P., Silva, L.A., Colodel, E.M. and Correa, F.R. (2014) Toxic Myopathy and Acute Hepatic Necrosis in Cattle Caused by Ingestions of Senna obtusifolia (Sicklepod; Coffee Senna) in Brazil. Toxicon, 92, 24-30. https://doi.org/10.1016/j.toxicon.2014.09.007

[29] Dionex (2009) Determination of Anthraquinones and Stilbeines in Giant Knotweed Rhizome by HPLC with UV Detection. Application Note 232.

[30] Norsworthy, J. and Oliveira, M. (2006) Sicklepod (Senna obtusifolia) Germination and Emergence as Affected by Environmental Factors and Seeding Depth. Weed Science, 54, 903-909. https://doi.org/10.1614/WS-06-070R2.1

[31] Han, Y.-S., van der Heijden, R. and Verpoorte, R. (2001) Biosynthesis of Anthraquinones in Cell Cultures of the Rubiaceae. Plant Cell Tissue and Organ Culture, 67, 201-220. https://doi.org/10.1023/A:1012758922713

[32] Vanderveer, V. (1935) Light Stable Insecticide. US Patent No. US2011428 A.

[33] Osman, N.M., Mohamed Ahmed, I.A. and Babiker, E.E. (2010) Fermentation and Cooking of Sicklepod (Cassia obtusifolia) Leaves: Changes in Chemical and Amino Acid Composition, Antinutrients and Protein Fractions and Digestibility. International Journal of Food Sciences and Technology, 45, 124-132.

https://doi.org/10.1111/j.1365-2621.2009.02111.x

[34] Ingweye, J.N., Kalio, G.A., Ubua, J.A. and Umoren, E.P. (2010) Nutritional Evaluation of Wild Sicklepod (Senna obtusifolia) Seeds from Obanliku, South-Eastern Nigeria. American Journal of Food Technology, 5, 1-12. https://doi.org/10.3923/ajft.2010.1.12

[35] Harborne, J.B. (1998) Phytochemical Methods: A Guide to Modern Techniques of Plant Analysis. 3rd Edition, Chapman and Hall, New York.

[36] Monteith, K.B. (2012) Nutritional Ecology of White-Tailed Deer: Assessment of an Index to Diet Quality and a Repellent. Master's Thesis, South Dakota State University, Brookings.

[37] Jacob, J.P., Mitaru, B.N., Mbugua, P.N. and Blair, R. (1996) The Feeding Value of Kenyan Sorghum, Sunflower Seed Cake and Sesame Seed Cake for Broilers and Layers. Animal Feed Science and Technology, 61, 41-46. https://doi.org/10.1016/0377-8401(96)00957-1

[38] Van Gorkom, B.A., de Vries, E.G., Karrenbeld, A. and Kleibeuker, J.H. (1999) Anthranoid Laxatives and Their Potential Carcinogenic Effects. Alimentary Pharmacology \& Therapeutics, 13, 443-452. https://doi.org/10.1046/j.1365-2036.1999.00468.x 